\title{
Gram per Milliliter per Milligram per Meter Squared
}

National Cancer Institute

\section{Source}

National Cancer Institute. Gram per Milliliter per Milligram per Meter Squared. NCI

Thesaurus. Code C119354.

A unit of concentration equal to grams per milliliter, divided by milligrams per meter squared. 